\title{
Hyperkähler manifolds and algebraic geometry
}

\author{
Kieran G. O'Grady* \\ Università di Roma "La Sapienza"
}

October 12004

\begin{abstract}
We survey some recent and less recent results on hyperkähler manifolds i.e. irreducible (holomorphic) symplectic manifolds. The point of view will be that of an algebraic (or complex) geometer.
\end{abstract}

\section{Introduction}

A compact Kähler surface $S$ is a $K 3$ if it is simply connected and it carries a global holomorphic symplectic form (i.e. the canonical bundle $K_{S}:=\wedge^{2} \Omega_{S}^{1}$ is trivial). An example: if $P\left(x_{0}, \ldots, x_{4}\right)$ is a homogeneous polynomial of degree 4 such that $\nabla P$ has no non-trivial zeroes then

$$
S=V(P):=\left\{\left[x_{0}, \ldots, x_{4}\right] \in \mathbb{P}_{\mathbb{C}}^{3} \mid P\left(x_{0}, \ldots, x_{4}\right)=0\right\}
$$

is a $K 3$ surface $^{1}$. These surfaces play an important rôle in the classification of Kähler manifolds and they have a very rich geometry. Thus it is natural to search for higher dimensional analogues of $K 3$ surfaces. A natural generalization of the definition of a $K 3$ is that of a Calabi-Yau: a compact Kähler manifold $X$ with trivial canonical bundle and $h^{0}\left(\Omega_{X}^{p}\right)=0$ for $0<p<\operatorname{dim} X^{2}$. Another generalization of the definition of a $K 3$ is that of an irreducible symplectic manifold: a simply connected compact Kähler manifold $X$ carrying a holomorphic symplectic form spanning $\Gamma\left(\Omega_{X}^{2}\right)$. It turns out that higher dimensional irreducible symplectic manifolds behave very much like $K 3$ 's in many respects. In these notes we will survey certain recent (and less recent) results which have been proved on these manifolds. First we explain why they are also known as hyperkähler manifolds. If $X$ is irreducible symplectic then by Yau's solution of Calabi's conjecture there exists a Riemannian metric $g$ on $X$ such that $(X, g)$ is an irreducible hyperkähler manifold i.e. the holonomy is isomorphic to the tautological representation of

$$
S p(r):=\left\{\phi: \mathbb{H}^{r} \rightarrow \mathbb{H}^{r} \mid \phi \text { right-linear and } \overline{\phi(\mathbf{v})}^{t} \cdot \phi(\mathbf{w})=\overline{\mathbf{v}}^{t} \cdot \mathbf{w}\right\}
$$

\footnotetext{
* Supported by Cofinanziamento MIUR 2003-2004

${ }^{1} S$ is simply connected by Lefschetz' Hyperplane section Theorem. The exact sequence $\left.\left.0 \rightarrow I_{S}\right|_{S} \rightarrow \Omega_{\mathbb{P}^{3}}^{1}\right|_{S} \rightarrow \Omega_{S}^{1} \rightarrow 0$ together with the isomorphisms $\left.I_{S}\right|_{S} \cong \mathcal{O}_{S}(-4)$ and $\wedge^{3} \Omega_{\mathbb{P}^{3}}^{1} \cong$ $\mathcal{O}_{\mathbb{P}^{3}}(-4)$ gives that $K_{S}$ is trivial.

${ }^{2}$ We do not require that $\pi_{1}(X)$ is trivial, only that $b_{1}(X)=0$. One shows that if $n=2$ the latter condition is equivalent to the former.
} 
on $\mathbb{H}^{r}$ where $4 r=\operatorname{dim}_{\mathbb{R}} X$. Conversely let $(M, g)$ be a compact irreducible hyperkähler manifold. Fix a point $p \in M$; left-multiplication by $\lambda=a i+b j+c k$ with $\lambda^{2}=-1$ on the tangent space $\Theta_{p} M$ is an endomorphism commuting with the holonomy group and hence gives $M$ the structure of a complex manifold $X_{\lambda}$ for which $g$ is the real part of a Kähler metric. Furthermore since $S p(r)=U\left(\mathbb{C}^{2 r}\right) \cap S p\left(\mathbb{C}^{2 r}\right)$ we get that $X_{\lambda}$ carries a holomorphic symplectic form spanning $\Gamma\left(\Omega_{X_{\lambda}}^{2}\right)$. It can be proved that $\pi_{1}(M)=\{1\}$ (see [2]) and hence $X_{\lambda}$ is an irreducible symplectic manifold. We will start our survey by giving the first examples of higher-dimensional $(\operatorname{dim}>2)$ irreducible symplectic manifolds that were ever constructed (Fujiki, Beauville): they give two distinct deformation classes in every (even) dimension greater than 2. In Section (3) we will review that part of the general theory that was developed roughly 25 years ago by Bogomolv, Fujiki and Beauville and we will state some of Huybrechts' recent theorems on the Kähler cone and surjectivity of the period map. These results give strong evidence in favour of the slogan higher-dimensional irreducible symplectic manifolds are analogues of K3 surfaces. In fact these manifolds, similarly to $K 3$ surfaces, are studied via periods of the symplectic form and the results of Section (3) are extensions to arbitrary dimension of theorems which had previously been proved to hold for $K 3$ surfaces. After that we review the many examples one encounters in algebraic geometry, mostly moduli spaces of sheaves on projective $K 3$ surfaces or abelian surfaces (following Mukai). These moduli spaces will also give us examples of interesting birational maps between irreducible symplectic manifolds. We will recall Huybrechts' beautiful Theorem which states that birational equivalent irreducible symplectic manifolds are deformation equivalent. In the following section we will give our construction of examples in dimensions 6 and 10 which are not deformations of the previously known ones. Every known higher-dimensional irreducible symplectic manifold is deformation equivalent to one of Beauville's examples or to one of our examples $^{3}$ - thus we know of 2 distinct deformation classes in every even dimension at least 4 with one extra deformation class in dimensions 6 and 10.

\section{$1.1 \quad$ Notation}

We will be working in the category of complex spaces or of complex algebraic varieties. Thus unless we specify otherwise a symplectic form is holomorphic.

\section{First higher dimensional examples}

Beauville constructed two families of irreducible symplectic manifolds in every even dimension greater than 2. The first family consists of Hilbert schemes of 0 -dimensional subschemes of a $K 3$, the second family consists of generalized Kummer manifolds. Members of distinct families are not deformation equivalent because they do not have the same Betti numbers.

\section{$2.1 \quad$ Hilbert schemes of $K 3$ 's}

Let $S$ be a $K 3$ surface: the Hilbert scheme (or Douady space) $S^{[n]}$ is the $2 n$-dimensional connected manifold parametrizing subschemes $Z \subset S$ of finite

\footnotetext{
${ }^{3}$ Kodaira proved roughly 40 years ago that any two $K 3$ surfaces are deformation equivalent.
} 
length ${ }^{4}$ equal to $n$. One forms a picture of $S^{[n]}$ by contemplating the cycle map

$$
\gamma_{n}: S^{[n]} \rightarrow S^{(n)}
$$

where $S^{(n)}$ is the symmetric product of $n$ copies of $S$. The map $\gamma_{n}$ is an isomorphism over $\left(S^{(n)}\right)_{0}$, the smooth locus of $S^{(n)}$, i.e. the subset parametrizing cycles $p_{1}+\cdots+p_{n}$ with pairwise distinct $p_{i}$ 's. The fibers of $\gamma_{n}$ over points of $\operatorname{sing}\left(S^{(n)}\right)$ are positive dimensional. Let's examine $S^{[2]}$ more closely. In this case we may avoid appealing to the theory of Hilbert schemes: simply define $S^{[2]}$ to be the blow-up of $S^{(2)}$ with center $\operatorname{sing}\left(S^{(2)}\right)$ and thus (2.1) is the blow-up map (this is Fujiki's construction of the first example of a higher dimensional irreducible symplectic manifold). Then $S^{[2]}$ is stratified according to the dimension of fibers of (2.1). There are two strata: the open stratum isomorphic to $\left(S^{(2)}\right)_{0}$ and the closed stratum isomorphic to the projectivization of the tangent bundle of $S$. The manifold $S^{[n]}$ is Kähler by a Theorem of Varouchas. One associate to a symplectic form $\sigma$ on $S$ a symplectic form $\sigma^{[n]}$ on $S^{[n]}$ as follows. Let $\pi_{i}: S^{n} \rightarrow S$ be the $i$-th projection. The 2 -form on $S^{n}$ given by $\sum_{i=1}^{n} \pi_{i}^{*} \sigma$ is symplectic and invariant under the action of the symmetric group hence it descends to a symplectic form on on $\left(S^{(n)}\right)_{0}$. Since $\gamma_{n}$ is an isomorphism over $\left(S^{(n)}\right)_{0}$ we get a symplectic form on $\gamma_{n}^{-1}\left(S^{(n)}\right)_{0}$; one verifies easily that this form extends to a symplectic form on $S^{[n]}$. We refer to [2] for the proof that $S^{[n]}$ is irreducible symplectic. The important Betti number $b_{2}\left(S^{[n]}\right)$ is computed as follows. One proves that the exceptional divisor $\gamma_{n}^{-1}\left(\operatorname{sing}\left(S^{(n)}\right)\right)$ is irreducible; from this one easily gets ${ }^{5}$ that

$$
b_{2}\left(S^{[n]}\right)=b_{2}\left(S^{(n)}\right)+1=b_{2}(S)+1=23, \quad n \geq 2 .
$$

\subsection{Generalized Kummer manifolds}

Let $T$ be a 2-dimensional complex torus ${ }^{6}$ and $\sigma$ be a symplectic form on $T$. Proceeding as in the case of $K 3$ surfaces one associates to $\sigma$ a symplectic form $\sigma^{[n+1]}$ on $T^{[n+1]}$. However $T^{[n+1]}$ is not irreducible symplectic. In fact consider the composition

$$
T^{[n+1]} \stackrel{\gamma_{n+1}}{\longrightarrow} T^{(n+1)} \stackrel{\zeta_{n+1}}{\longrightarrow} T,
$$

where $\gamma_{n+1}$ is the cycle map (see (2.1)) and $\zeta_{n+1}$ is the map defined by the addition law on $T$. By (2.3) we have $b_{1}\left(T^{[n+1]}\right) \geq b_{1}(T)=4$ and we also get that $\left(\zeta_{n+1} \circ \gamma_{n+1}\right)^{*}(\sigma)$ is a 2 -form independent of $\sigma^{[n+1]}$. On the other hand [2] it turns out that $K^{[n+1]}(T):=\left(\zeta_{n+1} \circ \gamma_{n+1}\right)^{-1}(0)$ is irreducible symplectic of dimension $2 n$. If $n=1$ this is the classical Kummer surface, a particular $K 3$. Considering the cycle map $\gamma_{n+1}$ one shows that

$$
b_{2}\left(K^{[n+1]}(T)\right)=b_{2}(T)+1=7, \quad n \geq 2 .
$$

\footnotetext{
${ }^{4}$ If the ideal sheaf of $Z$ is $I_{Z} \subset \mathcal{O}_{S}$ the length of $Z$ is the dimension of $\mathcal{O}_{Z}:=\mathcal{O}_{S} / I_{Z}$ as complex vector space.

${ }^{5}$ Thom-Hirzebruch's Index Theorem gives that $b_{2}(K 3)=22$.

${ }^{6} T=\mathbb{C}^{2} / \Lambda$ where $\Lambda \cong \mathbb{Z}^{4}$ is a discrete subgroup, i.e. it spans $\mathbb{C}^{2}$ over $\mathbb{R}$.
} 


\section{Periods}

Almost 50 years ago A. Weil [32] formulated a series of conjectures on moduli $^{7}$ and periods ${ }^{8}$ of $K 3$ surfaces. Most of the conjectures were proved in the following 20 years; the most celebrated result is the Global Torelli Theorem proved in the '70's by Piatechki-Shapiro and Shafarevich [27], Burns and Rapoport [7], Looijenga and Peters [20] (Friedman [11] gave a radically different proof). Roughly 20 years ago Beauville [20] started investigating periods of irreducible symplectic manifolds of arbitrary dimension (there had been a first attempt by Bogomolov [4]) and showed that periods of higher dimensionsal irreducible symplectic manifolds behave very much like those of $K 3$ 's. Recently Huybrechts $[15,16,17]$ proved many deep results on Kähler classes and moduli of irreducible symplectic manifolds. Huybrechts made heavy use of the period map, in particular periods of the twistor family $\left\{X_{\lambda}\right\}_{\lambda \in \mathbb{P}^{1}}$ described in Section (1). Notice that the twistor family exists because of the equivalence between irreducible symplectic manifolds and irreducible hyperkähler manifolds (i.e. thanks to Yau's solution of the Calabi conjecture).

\subsection{Deformations and the local period map}

Let $X$ be an irreducible symplectic manifold. Bogomolov [4] proved that deformations of $X$ are unobstructed. Thus there exists a proper submersive map $f: \mathcal{X} \rightarrow U$ where $U$ is a polydisc, $X \cong X_{0}:=f^{-1}(0)$ and any irreducible symplectic manifold whose complex structure is "close"to $X$ is isomorphic to $X_{t}:=f^{-1}(t)$ for some $t \in U$ (if $\operatorname{Aut}(X)$ is trivial then $t$ is unique, in general the set of such $t$ is at most countable). Furthermore the Kodaira-Spencer map

$$
\Theta_{U, 0} \stackrel{\kappa}{\longrightarrow} H^{1}\left(\Theta_{X}\right) \stackrel{\sim}{\longrightarrow} H^{1}\left(\Omega_{X}^{1}\right)
$$

is an isomorphism. (The second map of (3.1) is the isomorphism induced by contraction with a symplectic form.) We say that $f: \mathcal{X} \rightarrow U$ is a representative of $\operatorname{Def}(X)$ : in what follows we will feel free to shrink arbitrarily $U$ around 0 in other words we are mostly interested in the germs of $\mathcal{X}$ and $U$ at $X_{0}$ and 0 respectively. From (3.1) and the Hodge equality $b_{2}(X)=2 h^{2,0}(X)+h^{1,1}(X)$ we get that

$$
\operatorname{dim} U=b_{2}(X)-2 .
$$

Example 3.1. Let $S$ be $a K 3$ and $X=S^{[n]}$ with $n \geq 2$ : by (3.2)-(2.2) we have $\operatorname{dim} U=21$. On the other hand the deformation space of $S$ has dimension 20 by (3.2) and hence the generic deformation of $S^{[n]}$ is not of the form $(K 3)^{[n]}$. Similarly the generic deformation of a generalized Kummer manifold is not a generalized Kummer.

Now we define the period map. Since $U$ is contractible $\mathcal{X}$ is diffeomeorphic to $X \times U$ and hence for all $t \in U$ we have a well-defined integral isomorphism

$$
\phi_{t}: H^{2}(X) \stackrel{\sim}{\longrightarrow} H^{2}\left(X_{t}\right) .
$$

\footnotetext{
${ }^{7}$ Isomorphism classes.

${ }^{8}$ Integrals ("periods") of a symplectic form over integral 2-cycles.
} 
The local period map of $X$ is given by

$$
\begin{array}{ccc}
U & \stackrel{P_{X}}{\longrightarrow} & \mathbb{P}\left(H^{2}(X)\right) \\
t & \mapsto & \phi_{t}^{-1} H^{2,0}\left(X_{t}\right)
\end{array}
$$

By Griffiths's general results on the derivative of period maps the image of $d P_{X}(0)$ lies in the subspace

$$
\operatorname{Hom}\left(H^{0}\left(\Omega_{X}^{2}\right), H^{1}\left(\Omega_{X}^{1}\right)\right) \subset \operatorname{Hom}\left(H^{0}\left(\Omega_{X}^{2}\right), H^{2}(X) / H^{0}\left(\Omega_{X}^{2}\right)\right)
$$

and we have a natural identification of $d P_{X}(0)$ with the map

$$
\begin{array}{ccc}
H^{1}\left(\Theta_{X}\right) & \longrightarrow & \operatorname{Hom}\left(H^{0}\left(\Omega_{X}^{2}\right), H^{1}\left(\Omega_{X}^{1}\right)\right) \\
\theta & \mapsto & \operatorname{contr}(\cdot, \theta) .
\end{array}
$$

Since $H^{0}\left(\Omega_{X}^{2}\right)$ is spanned by a symplectic form $\sigma$ and contraction with $\sigma$ defines an isomorphism of vector-bundles $\Theta_{X} \stackrel{\sim}{\longrightarrow} \Omega_{X}^{1}$ we get that the above map is an isomorphism. Thus $d P_{X}(0)$ is injective and hence $P_{X}$ is an immersion of $U$ near 0 - this is the Local Torelli Theorem. By (3.6) (or by (3.2)) the image $P_{X}(U)$ is a smooth analytic subset of codimension 1 in $\mathbb{P}\left(H^{2}(X)\right)$.

\subsection{Beauville's quadratic form and Fujiki's constant}

Theorem 3.2. [(Beauville: Thm. (4) of [2])+(Fujiki: Thm. (4.7) of [12])] Let $X$ be an irreducible symplectic manifold of dimension $2 n$. There exist a positive rational number $c_{X}$ (Fujiki's constant) and an integral indivisible nondegenerate symmetric bilinear form $(,)_{X}$ on $H^{2}(X)$ (Beauville's form) of signature $\left(3, b_{2}(X)-3\right)$ such that the following hold:

(1) $\operatorname{Im}\left(P_{X}\right) \subset Q:=\left\{[\sigma] \in \mathbb{P}\left(H^{2}(X)\right) \mid(\sigma, \sigma)_{X}=0, \quad(\sigma, \bar{\sigma})_{X}>0\right\}$,

(2) $\int_{X} \alpha^{2 n}=c_{X} \cdot(\alpha, \alpha)_{X}^{n}$ for $\alpha \in H^{2}(X)$.

(3) $\left(\alpha, \alpha^{\prime}\right)_{X}=0$ if $\alpha \in H^{p, 2-p}(X), \alpha^{\prime} \in H^{p^{\prime}, 2-p^{\prime}}(X)$ with $p+p^{\prime} \neq 2$.

Proof. Let $\widetilde{F} \in S_{2 n} H^{2}(X)^{\vee}$ be the intersection form

$$
\widetilde{F}\left(\alpha_{1}, \ldots, \alpha_{2 n}\right):=\int_{X} \alpha_{1} \wedge \cdots \wedge \alpha_{2 n} .
$$

Let $[\alpha] \in \operatorname{Im}\left(P_{X}\right)$ and $\beta_{1}, \ldots, \beta_{n-1} \in H^{2}(X)$ be arbitrary; we claim that

$$
\widetilde{F}(\underbrace{\alpha, \ldots, \alpha}_{n+1}, \beta_{1}, \ldots, \beta_{n-1})=0 .
$$

In fact if $[\alpha]=P_{X}(t)$ then

$$
\widetilde{F}(\underbrace{\alpha, \ldots, \alpha}_{n+1}, \beta_{1}, \ldots, \beta_{n-1})=\int_{X_{t}} \phi_{t}(\alpha)^{n+1} \wedge \phi_{t}\left(\beta_{1}\right) \cdots \wedge \phi_{t}\left(\beta_{n-1}\right) .
$$

By definition of the period map we may represent $\phi_{t}(\alpha)$ by a (holomorphic) symplectic form and hence the integrand is represented by a sum of forms of type $(p, q)$ with $p \geq(2 n+2)$; since $\operatorname{dim} X=2 n$ these forms are identically zero 
and the integral vanishes. This proves (3.8). Let $F$ be the degree- $2 n$ polynomial defined by

$$
F(\gamma):=\widetilde{F}(\underbrace{\gamma, \ldots, \gamma}_{2 n}) .
$$

Setting $\beta_{1}=\cdots \beta_{n-1}=\alpha$ in (3.8) we get that $F$ vanishes on $\operatorname{Im}\left(P_{X}\right)$ : since $F$ is not identically zero ${ }^{9}$ it follows that the Zariski closure $^{10}$ of $\operatorname{Im}\left(P_{X}\right)$ in $\mathbb{P}\left(H^{2}(X)\right)$ is a proper subset of $\mathbb{P}\left(H^{2}(X)\right)$. On the other hand we know by Subsection (3.1) that $\operatorname{Im}\left(P_{X}\right)$ is a smooth connected analytic subset of codimension 1 in $\mathbb{P}\left(H^{2}(X)\right)$ and hence the Zariski closure of $\operatorname{Im}\left(P_{X}\right)$ is the set of zeroes of an irreducible non-zero homogeneous polynomial $A$. One verifies that $\operatorname{Im}\left(P_{X}\right)$ does not belong to a hyperplane, i.e. $\operatorname{deg} A \geq 2$. Since $F$ vanishes on $\operatorname{Im}\left(P_{X}\right)$ it vanishes also on $V(A)$ and hence by irreducibility of $A$ we have $F=F_{1} \cdot A$. If $n=1$ we have $2=\operatorname{deg} F=\operatorname{deg} F_{1}+\operatorname{deg} A \geq \operatorname{deg} F_{1}+2$ and hence $\operatorname{deg} F_{1}=0, \operatorname{deg} A=2$. Of course if $n=1$ the theorem is quite trivial: Beauville's form is the intersection form $F$ and Fujiki's constant is equal to 1. If $n=2$ we notice that (3.8) tells us that the partial derivatives of $F$ vanish on $\operatorname{Im}\left(P_{X}\right)$ and hence also on the zero-set of $A$. This implies that $A$ divides $F_{1}$ and hence

$$
F=F_{2} \cdot A^{2} .
$$

Thus $4=\operatorname{deg} F=\operatorname{deg} F_{2}+2 \operatorname{deg} A \geq \operatorname{deg} F_{2}+4$ and hence $\operatorname{deg} F_{2}=0, \operatorname{deg} A=2$. Equation (3.11) determines the constant $F_{2}$ and the quadratic form $A$ up to multiplicative factors; as is easily verified we can rescale $F_{2}$ and $A$ so that $A$ is integral, indivisible and $A(\sigma+\bar{\sigma}, \sigma+\bar{\sigma})>0$ for a (holomorphic) symplectic form $\sigma$. Let $(,)_{X}$ be the bilinear form defined by the "rescaled" quadratic polynomial $A$ and $c_{X}$ be the "rescaled" $F_{2}$. All the statements in the theorem hold by construction except possibly for the statement regarding the non-degeneracy and signature of $(,)_{X}$; this follows easily from the Hodge index Theorem. If $n>2$ one proceeds similarly; by (3.8) the partial derivatives of $F$ up to order $(n-1)$ vanish on $\operatorname{Im}\left(P_{X}\right)$ and hence also on the zero-set of $A$. Dividing $F$ successively by $A$ one gets that $F=F_{n} \cdot A^{n}$ where $F_{n}$ is a constant and $\operatorname{deg} A=2$. The rest of the argument is as in the case $n=2$.

A few comments:

(a) Let $U$ be a representative of $\operatorname{Def}(X)$ : by the Local Torelli Theorem (see the end of Subsection (3.1)) $P_{X}: U \rightarrow Q$ is an isomorphism onto an open subset of $Q$.

(b) The quantities $c_{X}$ and $(,)_{X}$ are uniquely characterized by Properties (1)(2) above and are invariant under deformation of complex structure; they are the main discreet invariants of $X$.

(c) Since $(,)_{X}$ is integral it gives $H^{2}(X ; \mathbb{Z})$ a structure of lattice ${ }^{11}$.

The discreet invariants of Beauville's examples are as follows. Let $S$ be a $K 3$ surface; then

$$
c_{S^{[n]}}=\frac{(2 n) !}{n ! 2^{n}}, \quad H^{2}\left(S^{[n]} ; \mathbb{Z}\right) \cong H^{\oplus 3} \oplus\left(-E_{8}\right)^{\oplus 2} \oplus(-2(n-1)), \quad n \geq 2
$$

\footnotetext{
${ }^{9}$ If $\omega$ is a Kähler class then $F(\omega)>0$.

${ }^{10}$ Common zeroes of all homogeneous polynomials vanishing on $\operatorname{Im}\left(P_{X}\right)$.

${ }^{11} \mathrm{~A}$ finitely generated free abelian group endowed with a non-degenerate integral symmetric bilinear form.
} 
where $H$ is the standard hyperbolic plane. Let $T$ be a 2-dimensional complex torus; then

$c_{K^{[n+1]}(T)}=\frac{(2 n) !}{n ! 2^{n}}(n+1), \quad H^{2}\left(K^{[n+1]}(T) ; \mathbb{Z}\right) \cong H^{\oplus 3} \oplus(-2(n+1)), \quad n \geq 2$.

\subsection{The Kähler cone and surjectivity of the period map}

We will state some of Huybrechts' recent results (with an improvement by Boucksom [5]); we refer to [18] for a very readable survey and of course to the original papers $[15,16,17,5]$. The first result is a projectivity criterion.

Proposition 3.3. [Projectivity criterion] An irreducible symplectic manifold $X$ is projective if and only if there exists $\alpha \in H_{\mathbb{Z}}^{1,1}(X)$ such that $(\alpha, \alpha)_{X}>0$.

Assume that $X$ is projective and that $L$ is an ample line bundle on $X$. Let $\sigma \in \Gamma\left(\Omega_{X}^{2}\right)$ be a symplectic form; then $\int_{X} c_{1}(L)^{2} \wedge(\sigma+\bar{\sigma})^{2 n-2}>0$, where $2 n=$ $\operatorname{dim} X$. Applying Items (2)-(3) of Theorem (3.2) we get that $\left(c_{1}(L), c_{1}(L)\right)_{X}>$ 0 . Thus the non-trivial part of the criterion is the sufficiency of the condition. The next result describes the Kähler cone $\mathcal{K}_{X} \subset H_{\mathbb{R}}^{1,1}(X)$ of Kähler classes. First we recall that the positive cone $\mathcal{C}_{X} \subset H_{\mathbb{R}}^{1,1}(X)$ is the connected component of $\left\{\alpha \in H_{\mathbb{R}}^{1,1}(X) \mid(\alpha, \alpha)_{X}>0\right\}$ containing $\mathcal{K}_{X}$.

Theorem 3.4. [Huybrechts [17]+Boucksom [5]] The Kähler cone $\mathcal{K}_{X}$ consists of those $\alpha \in \mathcal{C}_{X}$ such that $\int_{C} \alpha>0$ for all rational curves $C \subset X$.

Here a rational curve $C \subset X$ is the image of a non-constant map $\mathbb{P}^{1} \rightarrow$ $X$. A comment on the statement of the theorem. Demailly-Paun [9] have recently extended the Nakai-Moishezon theorem to the case of a compact Kähler manifold $X$, i.e. they proved that the Kähler cone is a connected component of the set of $\alpha \in H_{\mathbb{R}}^{1,1}(X)$ such that $\int_{Z} \alpha^{d}>0$ for all $d$-dimensional analytic subsets $Z \subset X$. Theorem (3.4) states that if $X$ is an irreducible symplectic manifold it suffices to test those $Z$ which are rational curves: in this respect $X$ really behaves like a $K 3$ surface. A comment on the proof: essential use is made of the twistor family $f: \mathcal{X} \rightarrow \mathbb{P}^{1}$ one can associate to an irreducible symplectic manifold together with the choice of a Kähler class - the complex structure on the fibers $X_{\lambda}:=f^{-1}(\lambda)$ is defined as in Section (1).

In order to formulate the last result we recall how to define the global period map. Choose a deformation class $\mathcal{D}$ of irreducible symplectic manifolds: thus there is a lattice $\Lambda$ with bilinear form $(,)_{\Lambda}$ such that for any $X \in \mathcal{D}$ the lattice $H^{2}(X ; \mathbb{Z})$ is isometric to $\Lambda$. The associated period space is

$$
Q_{\Lambda}:=\left\{[\sigma] \in \mathbb{P}(\Lambda \otimes \mathbb{C}) \mid(\sigma, \sigma)_{\Lambda}=0, \quad(\sigma, \bar{\sigma})_{\Lambda}>0\right\} .
$$

A marked manifold in $\mathcal{D}$ consists a couple $(X, \phi)$ where $X \in \mathcal{D}$ and $\phi: H^{2}(X ; \mathbb{Z}) \stackrel{\sim}{\rightarrow}$ $\Lambda$ is an isometry. To a marked manifold we associate its period

$$
P(X, \phi):=\phi_{\mathbb{C}}\left(H^{2,0}(X)\right) \in Q_{\Lambda} .
$$

The set of equivalence classes of marked manifolds in $\mathcal{D}$ is a (non-Hausdorff) analytic space $\mathcal{M}_{\mathcal{D}}$ and the period map $P: \mathcal{M}_{\mathcal{D}} \rightarrow Q_{\Lambda}$ is holomorphic.

Theorem 3.5. [Huybrechts [15]] Let $\mathcal{M}_{\mathcal{D}}^{0}$ be a connected component of $\mathcal{M}_{\mathcal{D}}$. The restriction of $P$ to $\mathcal{M}_{\mathcal{D}}^{0}$ is surjective onto $Q_{\Lambda}$.

Again the existence of the twistor family is essential for the proof. 


\section{More examples, birational maps}

We will present most of the known explicit constructions of irreducible symplectic manifolds. First the Fano variety of lines on a smooth cubic hypersurface in $\mathbb{P}^{5}$ - this example is due to Beauville and Donagi. Next we give the construction, due to Mukai, of a symplectic form on moduli spaces of stable sheaves on a projective surface $S$ with trivial canonical bundle ${ }^{12}$ and we recall the result (Mukai, Huybrechts-Göttsche, O'Grady, Yoshioka) stating that if such a moduli space is compact then it is an irreducible symplectic manifold ( $S$ a $K 3)$ or one of its "Bogomolov-Beauville factors" is ( $S$ an abelian surface). By this method one gets a very rich series of examples of irreducible symplectic varieties and also of interesting birational maps between them; we give explicit examples of Mukai flops, the symplest non-regular birational maps. We finish by stating Huybrechts' Theorem on birational irreducible symplectic manifolds.

\subsection{Lines on a cubic 4-fold}

Let $Y \subset \mathbb{P}^{5}$ be a smooth cubic hypersurface and $X:=F(Y)$ be the set of lines $L \subset X$. Thus $X$ is a closed subvariety of the Grassmannian $\mathbb{G r} r\left(1, \mathbb{P}^{5}\right)$. Beauville and Donagi [3] proved that $X$ is an irreducible symplectic manifold deformation equivalent to $(K 3)^{[2]}$. Let $\mathbb{G} r\left(1, \mathbb{P}^{5}\right) \hookrightarrow \mathbb{P}^{14}$ be the Plücker embedding. Thus we have $X \subset \mathbb{P}^{14}$; let $h:=c_{1}\left(\mathcal{O}_{X}(1)\right)$ be the first Chern class of the hyperplane bundle on $X$. One verifies [3] that $(h, h)_{X}=6$. The remarkable feature of Beauville-Donagi's example is the following: the family of $X=F(Y)$ one gets by letting $Y$ vary among all smooth cubic hypersurfaces is locally complete for deformations keeping the class $h$ of type $(1,1)$. In other words every small deformation of $X=F(Y)$ keeping $h$ of type $(1,1)$ is isomorphic to $X^{\prime}=F\left(Y^{\prime}\right)$ for some cubic hypersurface $Y^{\prime}$. I know of no other explicit locally complete family of higher-dimensional polarized irreducible symplectic varieties.

\subsection{Moduli spaces of sheaves}

Let $S$ be a projective surface. In general any natural algebraic structure on the set of isomorphism classes of vector-bundles on $S$ is not separated, i.e. not Hausdorff. In order to get separated moduli spaces one restricts to the class of $H$-stable vector-bundles, where $H$ is an ample divisor ${ }^{13}$ on $S$. In general moduli spaces of $H$-stable vector-bundles are not compact: in order to get compact moduli spaces one considers the larger class of $H$-semistable torsion-free sheaves and one introduces $\mathcal{S}$-equivalence, a relation which coincides with isomorphism for $H$-stable sheaves and is coarser than isomorphism for $H$-semistable non stable sheaves. Explicitely: a torsion-free sheaf $F$ is $H$-semistable if for all non-zero subsheaves $G \subset F$ we have

$$
\frac{1}{\operatorname{rk}(G)} \chi\left(G \otimes \mathcal{O}_{S}(m H)\right) \leq \frac{1}{\operatorname{rk}(F)} \chi\left(F \otimes \mathcal{O}_{S}(m H)\right) .
$$

If the inequality is strict whenever $G \neq F$ then $F$ is $H$-stable. A celebrated theorem of Gieseker and Maruyama states that the set of $\mathcal{S}$-equivalence classes of $H$-semistable torsion-free sheaves with fixed rank and Chern classes (in $H^{*}(S)$ )

\footnotetext{
${ }^{12}$ Thus $S$ is either a $K 3$ or an abelian surface.

${ }^{13}$ i.e. there exists an embedding $f: S \hookrightarrow \mathbb{P}^{n}$ with $f^{*} \mathcal{O}_{\mathbb{P}^{n}}(1) \cong \mathcal{O}_{S}(k H)$ for some $k>0$.
} 
has a natural structure of projective variety. Now let's assume that $K_{S}$ is trivial, i.e. that $S$ is a $K 3$ or an abelian surface. Given a positive $r \in \mathbb{N}, s \in \mathbb{Z}$ and $c_{1} \in H_{\mathbb{Z}}^{1,1}(S)$ we let $M\left(r, c_{1}, s\right)$ be the set of $\mathcal{S}$-equivalence classes of coherent pure $H$-semistable sheaves $F$ on $S$ with

$$
r k(F)=r, \quad c_{1}(F)=c_{1}, \quad \chi(F)= \begin{cases}r+s & \text { if } S \text { is a } K 3, \\ s & \text { if } S \text { is an abelian surface. }\end{cases}
$$

(To simplify notation we omit reference to $S, H$; however one must keep in mind that the moduli space depends both on $S$ and $H$.) Mukai [22] proved that the open subset $M^{s t}\left(r, c_{1}, s\right) \subset M\left(r, c_{1}, s\right)$ parametrizing stable sheaves is smooth and that if it is non-empty then

$$
\operatorname{dim} M^{s t}\left(r, c_{1}, s\right)=2-2 r s+c_{1}^{2} .
$$

Furthermore Mukai showed how to associate to a symplectic form $\sigma$ on $S$ a symplectic form $\sigma_{M}$ on $M^{s t}\left(r, c_{1}, s\right)$. We give the definition of $\sigma_{M}$ at a point $[F] \in M^{s t}\left(r, c_{1}, s\right)$ representing a locally-free sheaf, i.e. a vector-bundle. Since $F$ is a vector-bundle there is a canonical isomorphism ${ }^{14}$

$$
\Theta_{[F]} M\left(r, c_{1}, s\right) \cong H^{0,1}(\operatorname{End}(F)) .
$$

Given $\alpha, \beta \in H^{0,1}(\operatorname{End}(F))$ one sets

$$
\left\langle\sigma_{M}, \alpha \wedge \beta\right\rangle:=\int_{S} \sigma \wedge \operatorname{Tr}(\alpha \wedge \beta) .
$$

If $\left(r, c_{1}, s\right)$ and $H$ are suitably chosen then $M^{s t}\left(r, c_{1}, s\right)=M\left(r, c_{1}, s\right)$ and we may hope that $M\left(r, c_{1}, s\right)$ is an irreducible symplectic manifold.

Example 4.1. If $S$ is a $K 3$ surface then $M(1,0,1-n) \cong S^{[n]}$ : a sheaf is represented by a point of $M(1,0,1-n)$ if and only if it is isomorphic to $I_{Z}$ where $[Z] \in S^{[n]}$. If $S$ is an abelian surface then $M(1,0,-n) \cong S^{[n]} \times \operatorname{Pic}^{0}(S)$ : a sheaf is represented by a point of $M(1,0,-n)$ if and only if it is isomorphic to $I_{Z} \otimes L$ where $[Z] \in S^{[n]}$ and $[L] \in \operatorname{Pic}^{0}(S)$.

The example above suggests that $M\left(r, c_{1}, s\right)$ might be irreducible symplectic if $S$ is a $K 3$ but that if $S$ is an abelian surface we should first "reduce" $M\left(r, c_{1}, s\right)$. In fact one considers the map

$$
\begin{array}{ccc}
M\left(r, c_{1}, s\right) & \stackrel{\Phi}{\longrightarrow} & S \times P i c^{c_{1}}(S) \\
{[F]} & \mapsto & \left(\sum c_{2}^{r a t}(F),\left[\wedge^{r} F\right]\right)
\end{array}
$$

We explain our notation: $c_{2}^{r a t}(F) \in C H^{2}(S)$ is the 2-nd Chern class in the group of rational equivalence classes of 0-cycles on $S$ and $\sum: C H^{2}(S) \rightarrow S$ is induced by the addition law on $S,\left[\wedge^{r} F\right]$ is the isomorphism class of the line-bundle $\wedge^{r} F$. Assume that $\operatorname{dim} M\left(r, c_{1}, s\right) \geq 4$ : then $\Phi$ is submersive and any two of its fibers are isomorphic. Thus $M\left(r, c_{1}, s\right)^{0}:=\Phi^{-1}(a,[\xi])$ is well-defined up to isomorphism and by (4.3)

$$
\operatorname{dim} M\left(r, c_{1}, s\right)^{0}=-2-2 r s+c_{1}^{2} .
$$

\footnotetext{
${ }^{14}$ If $F$ is not locally-free replace $H^{0,1}(\operatorname{End}(F))$ by $\operatorname{Ext}^{1}(F, F)$.
} 
One verifies that the restriction of $\sigma_{M}$ to $M^{s t}\left(r, c_{1}, s\right)^{0}$ is symplectic. Now we can state the main result regarding $M\left(r, c_{1}, s\right)$ when $S$ is a $K 3$ and $M\left(r, c_{1}, s\right)^{0}$ when $S$ is an abelian surface under the hypothesis that $\left(r, c_{1}, s\right)$ and $H$ have been chosen so that $M^{s t}\left(r, c_{1}, s\right)=M\left(r, c_{1}, s\right)$.

Theorem 4.2. [[23, 13, 24, 30, 31]] Keep notation and hypotheses as above. If $S$ is a K3 then $M\left(r, c_{1}, s\right)$ is a deformation of $S^{[n]}$ where $2 n=2-2 r s+c_{1}^{2}$. If $S$ is an abelian surface and $\operatorname{dim} M\left(r, c_{1}, s\right) \geq 4$ then $M\left(r, c_{1}, s\right)^{0}$ is a deformation of $K^{[n+1]}(S)$ where $2 n=-2-2 r s+c_{1}^{2}$.

We notice that although the above moduli spaces belong to the same deformation class as Beauville's examples they are in general not isomorphic (and not birational) to Beauville's examples (recall Example (3.1)).

\subsection{Moduli spaces and Mukai flops}

We will examine a particular moduli space of sheaves on a $K 3$ surface. This will serve two purposes: it will show how one goes about proving Theorem (4.2) and it will introduce Mukai flops, the simplest non-regular birational maps between holomorphic symplecic manifolds. Let $S \subset \mathbb{P}^{3}$ be a smooth quartic surface, i.e. a hypersurface given by (1.1), and assume that $S$ contains a line $L$. Let $\ell:=c_{1}(L)$. We consider $M:=M(2, \ell,-1)$, where stability is with respect to $\mathcal{O}_{S}(1)$. As is easily checked $M=M^{s t}$. By the results of Mukai quoted in the preceding subsection we get that if $M$ is non-empty then it is a 4-dimensional smooth projective variety with a regular symplectic form. Let's show that $M$ is birational to $S^{[2]}$ : in particular this will prove that $M$ is irreducible symplectic.

Claim 4.3. Keeping notation as above, let $[F] \in M$. Then $h^{0}(F) \geq 1$.

Proof. Serre duality gives that $H^{2}(F) \cong \operatorname{Hom}\left(F, \mathcal{O}_{S}\right)^{\vee}$ and the last group vanishes by stability, hence $h^{2}(F)=0$. By definition of $M$ (see (4.2)) we have $\chi(F)=1$ and hence we get that $h^{0}(F) \geq 1$.

Let $\tau \in H^{0}(F)$ be non-zero. Then $\tau$ has isolated zeroes by stability of $F$ and hence $F$ fits into an exact sequence

$$
0 \rightarrow \mathcal{O}_{S} \stackrel{\tau}{\longrightarrow} F \longrightarrow I_{Z} \otimes \mathcal{O}_{S}(L) \rightarrow 0,
$$

where $I_{Z}$ is the ideal sheaf of a 0 -dimensional subscheme $Z \subset S$. From $\chi(F)=1$ we get that $\chi\left(I_{Z} \otimes \mathcal{O}_{S}(L)\right)=-1$ and hence $Z$ has length 2 . From this one easily gets that $M=M_{1} \amalg M_{2}$ where

$$
M_{i}:=\left\{[F] \in M \mid h^{0}(F)=i\right\} .
$$

By upper-semicontinuity of cohomology dimension we get that $M_{1}$ is open in $M$. One gets a regular map $M_{1} \rightarrow S^{[2]}$ by associating to $[F]$ the (unique) $Z$ appearing in Exact Sequence (4.8). One checks easily that this map gives an isomorphism

$$
f: M_{1} \stackrel{\sim}{\longrightarrow}\left(S^{[2]} \backslash L^{[2]}\right),
$$

where $L^{[2]} \subset S^{[2]}$ is the closed subset parametrizing subschemes of $L$. On the other hand we have an isomorphism

$$
M_{2} \cong\left(L^{[2]}\right)^{\vee} .
$$


(Explanation: $L^{[2]} \cong \mathbb{P}^{2}$ and $\left(L^{[2]}\right)^{\vee}$ is the dual plane.) Isomorphism (4.11) is defined as follows. To $[F] \in M_{2}$ we associate the set $R_{F}$ of $Z \subset S$ appearing in (4.8) as $\tau$ varies among $\left(H^{0}(F) \backslash\{0\}\right)$. One verifies easily that all $Z$ parametrized by $R_{F}$ are contained in $L$ and that $R_{F}$ is a line in $L^{[2]}$. From the above we get that $M_{1}$ is dense in $M$ and hence that $f$ defines a birational map $\bar{f}: M \cdots>S^{[2]}$. One checks that $\bar{f}$ is not regular. The inverse $\bar{f}^{-1}$ replaces $L^{[2]}$ by its dual plane $\left(L^{[2]}\right)^{\vee}$. This is an example of a Mukai flop, defined in general as follows. Let $X$ be an irreducible symplectic manifold with symplectic form $\sigma$. Assume that there exists a closed $Z \subset X$ of codimension $r$ and that we have a $\mathbb{P}^{r}$-fibration $\rho: Z \rightarrow B$. Let $p \in Z$ and $\mathbb{P}^{r}=\rho^{-1}(\rho(p))$ be the fiber of $\rho$ through $p$. The restriction of $\sigma$ to $Z$ is the pull-back of a 2 -form on $B$, hence contraction with $\sigma$ defines an isomorphism

$$
\Theta_{p} \mathbb{P}^{r} \cong\left(N_{Z / X}\right)_{p}^{\vee}
$$

Let $\tilde{X} \rightarrow X$ be the blow up of $Z$ and $E \subset \widetilde{X}$ be the exceptional divisor. From (4.12) we get an inclusion

$$
E \stackrel{\iota}{\hookrightarrow} Z \times_{B} Z^{\vee}
$$

where $\rho^{\vee}: Z^{\vee} \rightarrow B$ is the dual fibration of $\rho$ and $\operatorname{Im}(\iota)$ is the relative incidence subvariety consisting of couples $(p, H)$ with $\rho(p)=\rho^{\vee}(H)$ and $p \in H$. Thus in addition to the (blow-up) $\mathbb{P}^{r-1}$-fibration $\pi: E \rightarrow Z$ we have a dual $\mathbb{P}^{r-1}$-fibration $\pi^{\vee}: E \rightarrow Z^{\vee}$. By Nakano's contractibility criterion there is a morphism $\widetilde{X} \rightarrow X^{\vee}$ to a smooth $X^{\vee}$ contracting the fibers of $\pi^{\vee}$ and hence we have a non-regular birational map

$$
X \cdots>X^{\vee}
$$

which is an isomorphism outside $Z, Z^{\vee}$. This is a Mukai flop, see [22]. The complex manifold $X^{\vee}$ is simply connected and it has a symplectic form spanning the space of holomorphic 2-forms, hence if it is Kähler it is irreducible symplectic. In our example $X=M, X^{\vee}=S^{[2]}, r=2$ and $B=p t$. Markman [21] has introduced and studied so-called generalized Mukai flops. There are many natural birational maps between moduli spaces of sheaves on a $K 3$ or abelian surface: they are Mukai flops in low dimensions, in general they tend to be generalized Mukai flops.

\subsection{Huybrechts' Theorem}

Birational maps between irreducible symplectic manifolds have been studied intensely, see $[6,8,14,33]$. We single out Huybrechts' beautiful result.

Theorem 4.4. [Huybrechts [17]] Let $X, Y$ be birational (bimeromorphic) irreducible symplectic manifolds. Then $X, Y$ are deformation equivalent.

The theorem above should be compared to theorems of Batyrev [1] and Denef-Loeser [10] stating that birational manifolds with trivial canonical bundles have the same Betti numbers, respectively Hodge numbers; however birational CY's need not be deformation equivalent, in fact they may not be homeomorphic. As in the proof of Theorems (3.4)-(3.5) a key rôle is played by the Twistor family. 


\section{New deformation classes}

We will sketch our construction [25, 26] of 6- and 10-dimensional irreducible symplectic manifolds which are not deformation equivalent to Beauville's examples. Let $S$ be a $K 3$ or abelian surface with an ample divisor $H$. We consider the moduli space $M(2,0,-2)$. This is a typical example in which $M^{s t}(2,0,-2) \neq M(2,0,-2)$; if $S$ is a $K 3$ the sheaf $I_{Z} \oplus I_{W}$ where $[Z],[W] \in S^{[2]}$ is a semistable non-stable sheaf parametrized by $M(2,0,-2)$, if $S$ is a torus the sheaf $\left(I_{p} \otimes L\right) \oplus\left(I_{p^{\prime}} \otimes L^{\prime}\right)$ where $p, p^{\prime} \in S$ and $[L],\left[L^{\prime}\right] \in P i c^{0}(S)$ is a semistable non-stable sheaf parametrized by $M(2,0,-2)$. If $H$ is chosen "generically" these are precisely the semistable non-stable sheaves parametrized by $M(2,0,-2)$ and their moduli sweep out the singular locus of $M(2,0,-2)$. I was able to construct a symplectic desingularization $\pi: \widetilde{M}(2,0,-2) \rightarrow M(2,0,-2)$ with $\widetilde{M}(2,0,-2)$ projective; symplectic means that $\pi^{*} \sigma_{M}$ extends to a symplectic form on $\widetilde{M}(2,0,-2)$. If $S$ is a torus set $\widetilde{M}(2,0,-2)^{0}:=\pi^{-1}\left(M(2,0,-2)^{0}\right)$; by (4.3) and (4.7) we have $\operatorname{dim} \widetilde{M}(2,0,-2)=10$ and $\widetilde{M}(2,0,-2)^{0}=6$. We proved that if $S$ is a $K 3$ then $\widetilde{M}(2,0,-2)$ is irreducible symplectic and

$$
b_{2}(\widetilde{M}(2,0,-2)) \geq 24 .
$$

This shows that $\widetilde{M}(2,0,-2)$ belongs to a new deformation class of 10-dimensional irreducible symplectic manifolds because for the standard Beauville examples $b_{2}$ is either 7 or 23 . We proved also that if $S$ is a torus then $\widetilde{M}(2,0,-2)^{0}$ is irreducible symplectic and

$$
b_{2}\left(\widetilde{M}(2,0,-2)^{0}\right)=8 .
$$

Thus $\widetilde{M}(2,0,-2)^{0}$ belongs to a new deformation class of 6 -dimensional irreducible symplectic manifolds. A few comments on the proof. The symplectic desingularization is obtained by first following Kirwan's procedure that gives (partial) desingularizations of GIT quotients whenever there are semistable nonstable orbits and then by contracting an extremal ray - see also Kaledin and Lehn [19] for an approach which avoids the contraction. The hardest part of the proof consists in showing that $\widetilde{M}(2,0,-2)$ (when $S$ is a $K 3$ ) or $\widetilde{M}(2,0,-2)^{0}$ (when $S$ is an abelian surface) is irreducible symplectic and that (5.2) holds Inequality (5.1) for $S$ a $K 3$ is straightforward. To explain why this is so let's take a step backwards: Theorem (4.2) is proved by showing that for a suitable choice of $(S, H)$ the moduli space is isomorphic to $(K 3)^{[n]}$ (birational suffices by Huybrechts' Theorem (4.4)) and this is also the quickest method for showing that $M$ (or $M^{0}$ ) is irreducible symplectic. In studying $\widetilde{M}$ and $\widetilde{M}^{0}$ we need to proceed differently: for the moment being we have no description of $\widetilde{M}$ (or $\widetilde{M}^{0}$ ) other than as a moduli space. Applying Lefschetz' Hyperplane Section Theorem we can describe quite explicitely a certain subset of $\widetilde{M}$ (or $\widetilde{M}^{0}$ ) whose low-dimensional topology resembles that of the mysterious variety we are studying. Examining this subset we are able to show that the mysterious variety is irreducible symplectic and also that (5.2) holds when $S$ is a torus. The basic discreet invariants of $\widetilde{M}^{0}$ have been computed recently by Rapagnetta [28]. In the same paper Rapagnetta also proved that the topological Euler characteristic of $\widetilde{M}^{0}$ is equal to 1920 . The question that naturally arises is whether one can 
generalize the above construction to produce other deformation classes of irreducible symplectic manifolds. In [25] we studied $M(2,0,2-2 k)$ for $S$ a $K 3$ and any $k \geq 2$ (if $k<2$ we get nothing interesting). If $H$ is chosen generically the semistable non-stable sheaves are represented by $I_{Z} \oplus I_{W}$ where $[Z],[W] \in S^{[k]}$ and their moduli sweep out the singular locus of the $(8 k-6)$-dimensional space $M(2,0,2-2 k)$. The singularities of $M(2,0,2-2 k)$ for $k>2$ differ from those of $M(2,0,-2)$ : in [25] we constructed a projective symplectic partial desingularization $\widetilde{M}(2,0,2-2 k) \rightarrow M(2,0,2-2 k)$ which is an actual desingularization only when $k=2$. Recently Kaledin and Lehn [19] proved that $M(2,0,2-2 k)$ has no symplectic resolution if $k>2$. Of course there are many other moduli spaces $M\left(r, c_{1}, s\right)$ for which $M^{s t}\left(r, c_{1}, s\right) \neq M\left(r, c_{1}, s\right)$. However $M\left(2 r^{\prime}, 2 c_{1}^{\prime}, 2 s^{\prime}\right)$ with $\left(r^{\prime}, c_{1}^{\prime}, s^{\prime}\right)$ an indivisible vector in $\left(\mathbb{Z} \oplus H^{2}(S ; \mathbb{Z}) \oplus \mathbb{Z}\right)$ is deformation equivalent to $M(2,0,2-2 k)$ for an approppriate $k$ and hence we will get nothing new. In general it looks unlikely that we will find new deformation classes of irreducible symplectic manifolds by desingularizing moduli spaces $M\left(r, c_{1}, s\right)$.

\section{References}

[1] V. Batyrev, Birational Calabi-Yau n-folds have equal Betti numbers, New trends in algebraic geometry (Warwick 1996), London Math. Soc. Lecture Note Ser. 264, CUP, 1999, pp. 1-11.

[2] A. Beauville, Variétes Kähleriennes dont la premiére classe de Chern est nulle, J. Differential geometry 18, 1983, pp. 755-782.

[3] A. Beauville - R. Donagi, La variétés des droites d'une hypersurface cubique de dimension 4. C. R. Acad. Sci. Paris Sér. I Math. 301, 1985, pp. 703-706.

[4] F. Bogomolov, Hamiltonian Kählerian manifolds, Soviet Math. Dokl. 19 (1978), 1979, pp. 1462-1465.

[5] S. Boucksom, Le cône kählérien d'une varieté hyperkählérienne, C. R. Acad. Sci. Paris 333, 2001, pp. 935-938.

[6] D. Burns - Y. Hu - T. Luo, HyperKhler Manifolds and Birational Transformations in dimension 4, arXiv:math.AG/0004154.

[7] D. Burns - M. Rapoport, On the Torelli problem for Kählerian K3 surfaces, Ann. scient. Éc. Norm. Sup. 8 (1975), pp. 235-274.

[8] K. Cho - Y. Miyaoka - N. Shepherd-Barron, Characterizations of projective space and applications to complex symplectic manifolds, Higher dimensional birational geometry (Kyoto, 1997), Adv. Stud. Pure Math. 35, Math. Soc. Japan, 2002, pp. 1-88.

[9] J. P. Demailly - M. Paum(?), Numerical characterization of the Khler cone of a compact Khler manifold, math.AG/0105167.

[10] J. Denef - F. Loeser, Germs of arcs on singular algebraic varieties and motivic integration, Invent. Math. 135, 1999, pp. 201-232.

[11] R. Friedman, A new proof of the global Torelli theorem for K3 surfaces, Ann. of Math. 120, 1984, pp. 237-269. 
[12] A. Fujiki, On the de Rham Cohomology Group of a Compact Kähler Symplectic Manifold, Adv. Studies in Pure Math. 10, Algebraic Geometry, Sendai 1985, 1987, pp. 105-165.

[13] L. Göttsche - D. Huybrechts, Hodge numbers of moduli spaces of stable bundles on K3 surfaces, Internat. J. Math. 7, 1996, pp. 359-372.

[14] Y. Hu - S.-T. Yau, HyperKähler manifolds and birational transformations, Adv. Theor. Math. Phys. 6, 2002, pp. 557-574.

[15] D. Huybrechts, Compact hyper-Khler manifolds: basic results, Invent. Math. 135, 1999, pp. 63-113.

[16] D. Huybrechts, Erratum: "Compact hyper-Khler manifolds: basic results"[Invent. Math. 135 (1999), no. 1, 63-113], Invent. Math. 152, 2003, pp. 209-212.

[17] D. Huybrechts, The Kähler cone of a compact hyperkähler manifold, Math. Ann. 326, 2003, pp. 499-513.

[18] D. Huybrechts, Compact hyperkähler manifolds, Calabi-Yau manifolds and related geometries (Nordfjordeid 2001), Universitext, Springer, Berlin, 2003, pp. 161-225.

[19] D. Kaledin - M. Lehn, Local structure of hyperkähler singularities in O'Grady examples, arXiv:math.AG/0405575.

[20] C. Peters - E. Looijenga, Torelli theorems for Khler K3 surfaces, Compositio Math. 42, 1980/81, 145-186.

[21] E. Markman, Brill-Noether duality for moduli spaces of sheaves on K3 surfaces, J. Algebraic Geom. 10, 2001, pp. 623-694.

[22] S. Mukai, Symplectic structure of the moduli space of sheaves on an abelian or K3 surface, Invent. math 77, 1984, pp. 101-116.

[23] S. Mukai, On the moduli space of bundles on K3 surfaces, I, Vector Bundles on Algebraic Varieties, TIFR, Bombay, O.U.P., 1987, pp. 341-413.

[24] K. G. O'Grady, The weight-two Hodge structure of moduli spaces of sheaves on a K3 surface, J. Algebraic Geom. 6, 1997, pp. 599-644.

[25] K. G. O'Grady, Desingularized moduli spaces of sheaves on a K3, J. fur die reine und angew. Math. 512, 1999, pp. 49-117.

[26] K. G. O'Grady, A new six-dimensional irreducible symplectic variety, J. Algebraic Geom. 12 (2003), pp. 435-505.

[27] I. Piatechki-Shapiro, I.R. Shafarevich A Torelli theorem for algebraic surfaces of type K3, Math. USSR Izvestija 5 (1971) pp. 547-588.

[28] A. Rapagnetta, Topological invariants of O'Grady's six dimensional irreducible symplectic variety, arXiv:math.AG/0406026.

[29] J. Varouchas, Sur l'image d'une variété kählérienne compacte, Fonctions de plusieurs variables complexes, V (Paris, 1979-1985), Springer LNM 1188, 1986, pp. 245-259.

[30] K. Yoshioka, Some examples of Mukai's reflections on K3 surfaces, J. Reine Angew. Math. 515, 1999, pp. 97-123. 
[31] K. Yoshioka, Moduli spaces of stable sheaves on abelian surfaces, Math. Ann. 321, 2001, pp. 817-884.

[32] A. Weil, Final report on contract AF 18(603)-57, André Weil - Collected papers, vol. II, Springer, 1979, pp. 393-395.

[33] J. Wierzba - J. Wiśniewski Small contractions of symplectic 4-folds, Duke Math. J. 120, 2003, pp. 65-95.

Kieran G. O'Grady

Università di Roma "La Sapienza",

Dipartimento di Matematica "Guido Castelnuovo",

Piazzale Aldo Moro n. 5, 00185 Rome, Italy,

e-mail: ogrady@mat.uniroma1.it. 